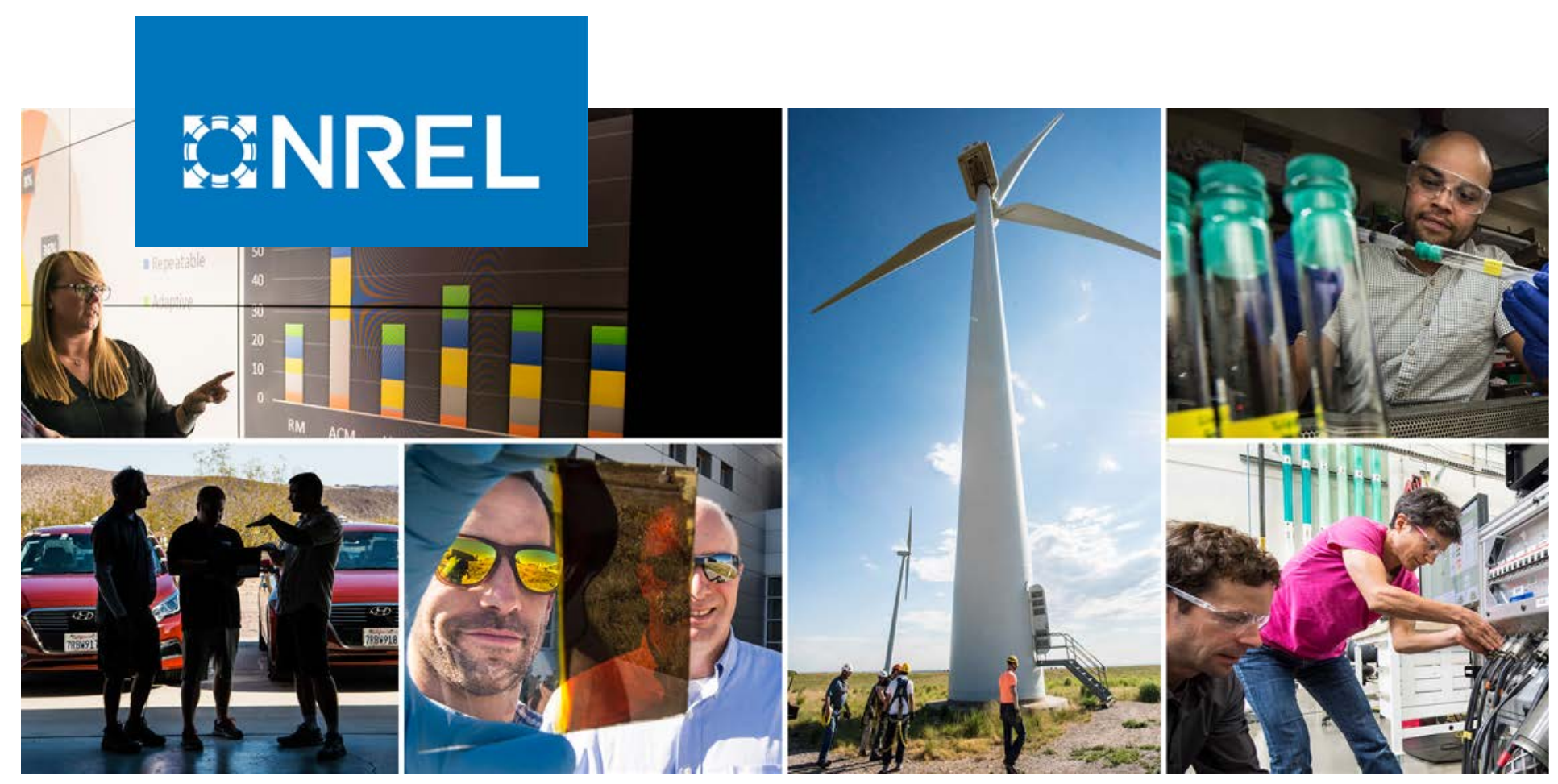

\title{
A Primer on FERC Order No. 2222: Insights for International Power Systems
}

Ella Zhou, David Hurlbut, and Kaifeng Xu

National Renewable Energy Laboratory

NREL is a national laboratory of the U.S. Department of Energy

Office of Energy Efficiency \& Renewable Energy

Operated by the Alliance for Sustainable Energy, LLC

This report is available at no cost from the National Renewable Energy Laboratory (NREL) at www.nrel.gov/publications.
Technical Report

NREL/TP-5C00-80166

September 2021 


\title{
GNREL
}

\section{A Primer on FERC Order No. 2222: Insights for International Power Systems}

\author{
Ella Zhou, David Hurlbut, and Kaifeng Xu
}

National Renewable Energy Laboratory

\section{Suggested Citation}

Zhou, Ella, David Hurlbut, and Kaifeng Xu. 2021. A Primer on FERC Order No. 2222: Insights for International Power Systems. Golden, CO: National Renewable Energy Laboratory. NREL/ TP-5C00-80166. https://www.nrel.gov/docs/fy21osti/80166.pdf.

NREL is a national laboratory of the U.S. Department of Energy Office of Energy Efficiency \& Renewable Energy Operated by the Alliance for Sustainable Energy, LLC

This report is available at no cost from the National Renewable Energy Laboratory (NREL) at www.nrel.gov/publications.

Contract No. DE-AC36-08GO28308
Technical Report

NREL/ TP-5C00-80166

September 2021

National Renewable Energy Laboratory 15013 Denver West Parkway Golden, CO 80401 303-275-3000 • www.nrel.gov 


\section{NOTICE}

This work was authored by the National Renewable Energy Laboratory, operated by Alliance for Sustainable Energy, LLC, for the U.S. Department of Energy (DOE) under Contract No. DE-AC36-08GO28308. Funding provided by Children's Investment Fund Foundation. The views expressed herein do not necessarily represent the views of the DOE or the U.S. Government.

This report is available at no cost from the National Renewable Energy Laboratory (NREL) at www.nrel.gov/publications.

U.S. Department of Energy (DOE) reports produced after 1991 and a growing number of pre-1991 documents are available free via www.OSTI.gov.

Cover Photos by Dennis Schroeder: (clockwise, left to right) NREL 51934, NREL 45897, NREL 42160, NREL 45891, NREL 48097, NREL 46526.

NREL prints on paper that contains recycled content. 


\section{List of Acronyms and Abbreviations}

DER

FERC

$\mathrm{GW}_{\mathrm{ac}}$

ISO

RTO distributed energy resource

U.S. Federal Energy Regulatory Commission

gigawatts-alternating current

independent system operator

regional transmission organization 


\section{Table of Contents}

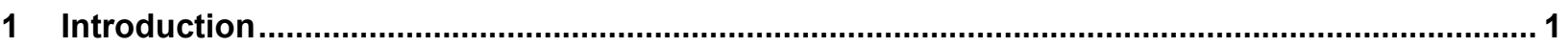

2 Implementation of Order No. 2222 ....................................................................................... 2

3 Values of Distributed Energy Resources: What motivated Order No. 2222? ............................4

4 Conclusions and Lessons for International Power Markets ...................................................... 5

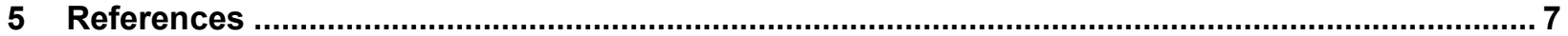

\section{List of Figures}

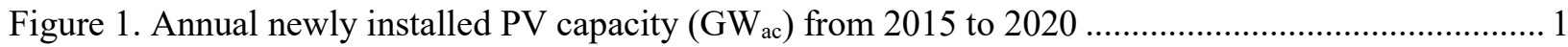

Figure 2. Illustration of DER aggregation in the RTO/ISO network ...................................................... 3 


\section{Introduction}

Distributed energy resource (DER) refers to "any resource located on the distribution system, any subsystem thereof or behind a customer meter", which may include, but not limited to, "electric storage resources, distributed generation, demand response, energy efficiency, thermal storage, and electric vehicles and their supply equipment" (FERC 2020b). Up to now, these technologies have provided individual benefits to the end-use customers who own them. U.S. Federal Energy Regulatory Commission (FERC) Order No. 2222 opens a path for new and potentially increased value, one that is based on intelligently combining many DERs into a single virtual resource that can reduce costs for the bulk power system. Unlike a small individual DER, an aggregation of DERs submitted as a single offer may participate in the capacity, energy, and ancillary service markets operated by regional transmission organizations (RTOs) and independent system operators (ISOs). In these markets, the types of DER technologies that make up an aggregation are not as important as the total number of megawatts (up or down) that the aggregation can provide dependably when the RTO calls for it. The expectation is that the combined value to the electric grid of the aggregated DERs is greater than the apportioned value individual DER owners would get by marketing or controlling their resources separatelyadditional value that could be shared among the individuals participating in the DER aggregation.

Deployment of DERs, in particular distributed solar photovoltaics (PV), has grown rapidly in the United States. Of the 73.8 gigawatts-alternating current $\left(\mathrm{GW}_{\mathrm{ac}}\right)$ of installed PV in the United States by the end of 2020, 27.7 GW were distributed PV in the commercial, industrial, and residential sectors (Figure 1). In some states, such as Texas, North Carolina, Florida, and Georgia, new distributed PV has outpaced the growth of utility-scale PV (Feldman and Margolis 2021).

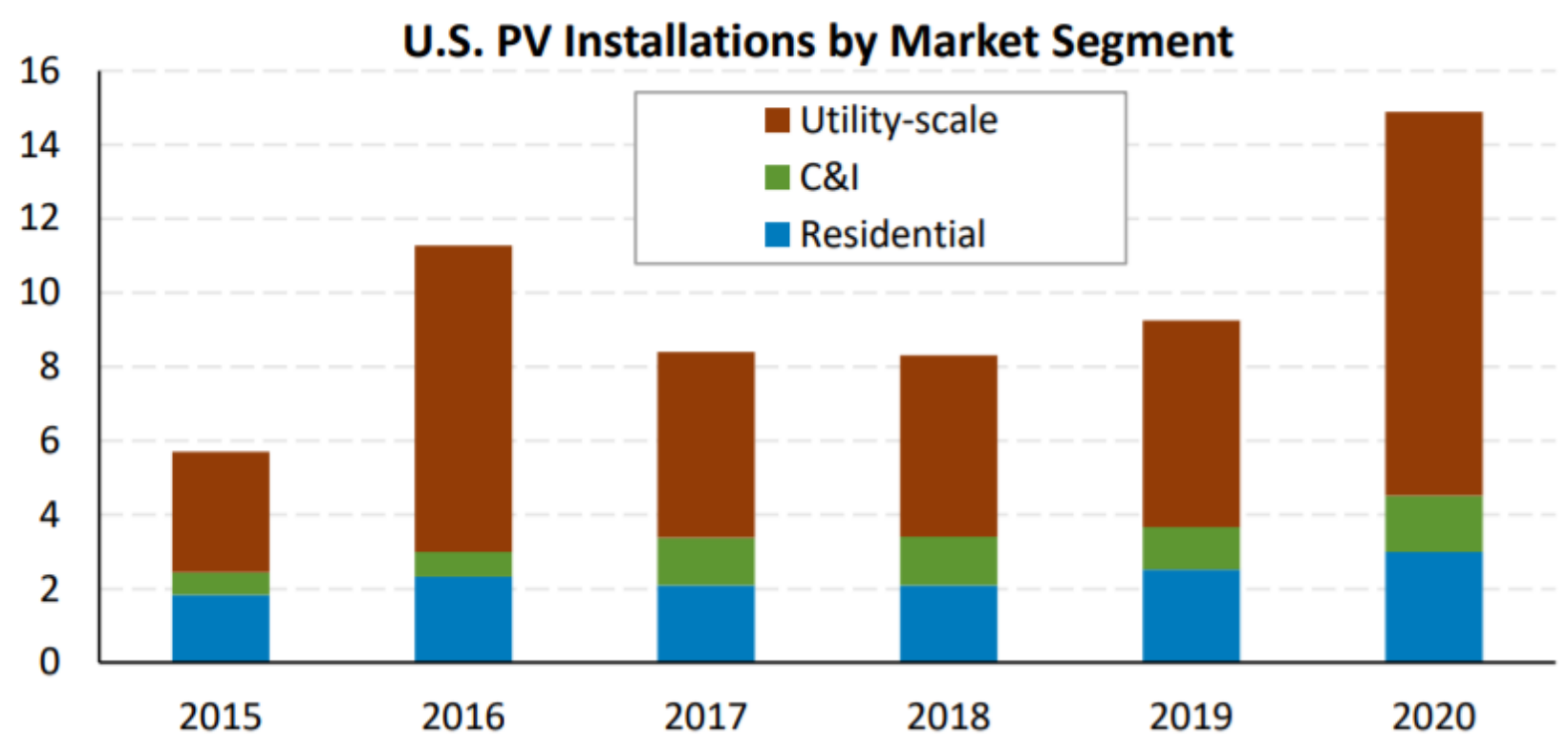

Figure 1. Annual newly installed PV capacity ( $G W_{a c}$ ) from 2015 to 2020

$C \& I=$ commercial $\&$ industrial 
Other important types of DERs include demand response, electric vehicles, and battery storage. About $31.5 \mathrm{GW}$ and $27.5 \mathrm{GW}$ of demand resources were enrolled in retail and wholesale demand resource markets, respectively, as of 2017 (FERC 2020a). Whereas traditional demand response focuses on peak demand reduction (also called "load shedding"1), the current definition of demand response resources has expanded to include flexible loads that can be shifted and modulated (Neukomm, Nubbe, and Fares 2019).

Load shift refers to the end use's ability to change the timing of electricity consumption, which might lead to using more electricity in lower-priced periods. Flexible loads that can be modulated can balance power supply and demand or reactive power draw within seconds based on automatic generation control signals (Neukomm, Nubbe, and Fares 2019). A recent California demand response study finds that $1 \%-2 \%$ of typical daily CAISO consumption in 2020 could be shifted over the course of the day (Gerke, Gallo, and Smith 2020). Electrification, especially electric vehicle deployment, has the potential to greatly expand the demand response available (Gerke, Gallo, and Smith 2020; Mai et al. 2018).

Driven by cost declines, state actions, ${ }^{2}$ and a greater need for grid balancing and resiliency, battery energy storage is also gaining momentum in the DER market (EIA 2020; Feldman and Margolis 2021). A Wood Mackenzie (2020) study estimates the cumulative distributed battery energy storage capacity will grow by 6,309 MW from 2021 to 2025, which represents a 573\% growth from the previous 5 years.

Energy efficiency is also a form of DER. For example, 2,900 MW of energy efficiency in the ISO-New England service area participated in the capacity market in 2019, and that amount is expected to increase dramatically over the next 10 years (ISO-NE 2020).

Due to the rapid development and huge potential of DERs, wholesale power markets need a special framework to access the values DERs can provide.

\section{Implementation of Order No. 2222}

FERC Order No. 2222 instructs RTOs and ISOs to allow DER aggregations to participate directly in the wholesale markets and establish a new category of market participants - namely DER aggregators. A DER aggregator is "an entity that aggregates one or more DER for purposes of participation in the capacity, energy and ancillary service markets of the regional transmission operators and independent system operators" (FERC 2020b). It serves to combine the DERs that previously might have been deployed individually (Horowitz et al. 2019).

The RTOs/ISOs would need to allow DER aggregators to register their resources under "one or more participation models that accommodate the physical and operational characteristics" of the DER aggregation (FERC 2020b) because existing generation models and demand response models might limit the operation of DERs and the services they may be eligible to provide. This

\footnotetext{
${ }^{1}$ During such demand response event, the amount by which the load is reduced during the event period, compared to what the load would have been under normal operation is referred to as load shed (FERC 2009).

${ }^{2}$ For example, California's Assembly Bill 2514 mandates the investor-owned utilities to procure energy storage across the transmission, distribution, and customer levels.
} 
situation requires the RTOs/ISOs to modify existing participation models to address their respective limitations for DERs, to create one or more new participation models, or to use a mix of existing and new participation models.

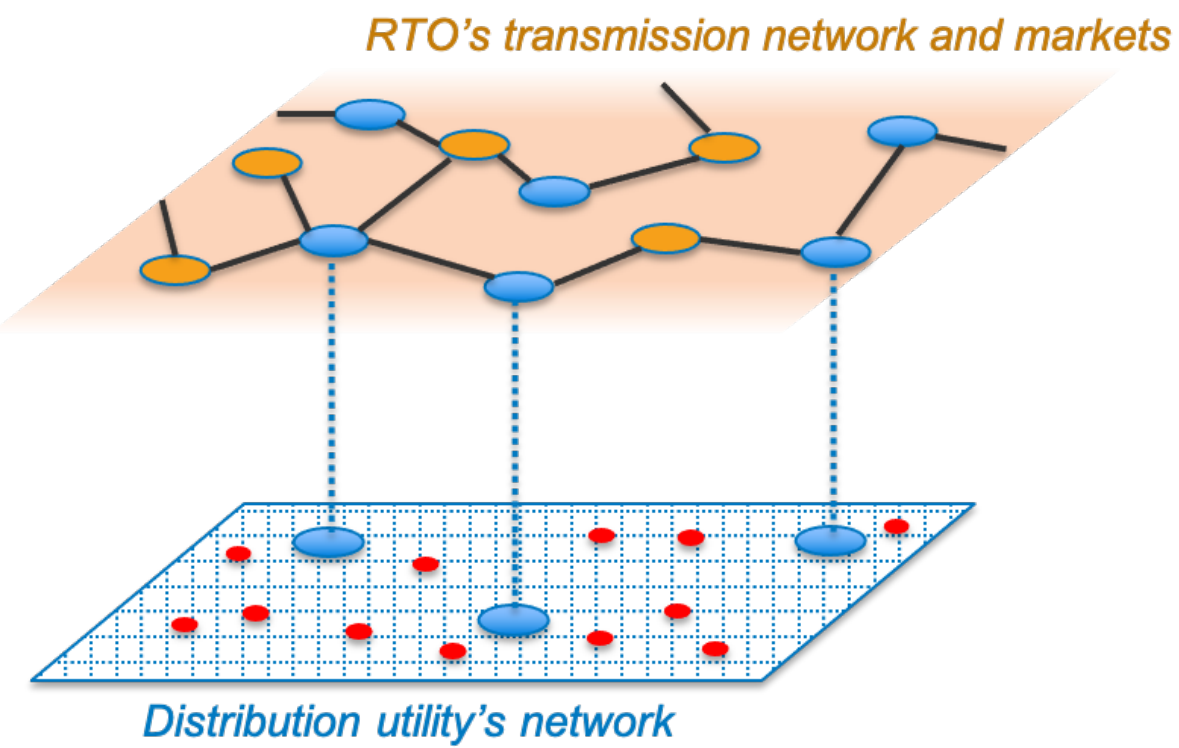

\section{- Aggregator's DER participants Load node Generator node}

Figure 2. Illustration of DER aggregation in the RTO/ISO network

Many directives in the Order are aimed at enhancing market competition. An RTO or ISO has some flexibility in how it designs its rules as long as it does not unnecessarily limit DER market entry. For example, Order No. 2222 allows aggregation of heterogenous technologies, including emerging and future technologies. It allows the RTO/ISO to set a minimum size requirement no larger than $100 \mathrm{~kW}$ and allows a single DER to be its own DER aggregation. It allows aggregation at a single node or multiple nodes and across broad geographic footprints (Figure 2).

At the same time, the RTO/ISO needs to prevent double-counting DERs. Some utilities offer local DER programs to their customers. While the DERs might be eligible for both the utility's demand response program and DER aggregation in the RTO/ISO, the same real-time DER response may not count in both programs.

Order No. 2222 includes directives on information, data, metering, and telemetry requirements. The DER aggregator, not individual DER in the aggregation, is the point of contact with the RTO/ISO. As such, the aggregator is responsible for "managing, dispatching, metering, and settling" the individual DER in its aggregation (FERC 2020b). Therefore, the information and data requirement covers the whole portfolio, even though the aggregator would need to list the individual resources in its aggregation along with any other necessary information as required by the RTO/ISO (FERC 2020b). The metering requirements are needed to provide settlement and performance data, and/or to prevent double counting of services. The telemetry requirements are 
necessary for the RTO/ISO to have situation awareness ${ }^{3}$ for dispatching and managing the DER aggregation, and such visibility is critical to maintaining the reliability of the bulk power system. The standardized collection of information and data and aligned communication protocols would be crucial for ensuring interoperability of the system with plentiful distinctive DER aggregations (McGranaghan et al. 2016).

Another important directive in Order No. 2222 is establishing market rules on coordination between the RTO/ISO, DER provider, distribution utility, and the relevant electric retail regulatory authorities. The physical interconnection of DERs falls under state or local jurisdiction in the United States. Therefore, the RTO/ISO needs to coordinate with state regulatory authorities to ensure the state policy and the RTO/ISO policy are aligned. The RTO/ISOs must incorporate a process to allow the distribution utility's review of the individual DER, in which the distribution utility would determine (1) whether each DER's interconnection can physically participate in an aggregation and (2) that the participation of each DER will not create a distribution network reliability or safety issue. The RTO/ISO would need to continue operational coordination with distribution utilities; address data flows and communication between the RTO/ISO, DER provider, and the distribution utility; and establish protocols and processes that allow distribution utilities to override the RTO/ISO dispatch of a DER aggregation on the operating day if such action were needed to maintain the reliability and safety of the distribution network. Relevant electric retail regulatory authorities can voluntarily participate in coordinating the DER aggregators in the wholesale market; and Order No. 2222 outlines some potential roles for them, including roles in the interconnection processes and in dispute resolution between the DER provider and distribution utility.

\section{Values of Distributed Energy Resources: What motivated Order No. 2222?}

FERC Order No 2222 represents an effort to ensure utility regulation keeps up with DER capabilities, which are improving quickly. These new capabilities bring more options for achieving public policy goals, such as applying just and reasonable rates for customers and decarbonizing the power sector.

Perhaps the largest driver for Order No. 2222 is the desire to improve wholesale power markets so that price outcomes are more just and more reasonable. Price-responsive demand is an important part of a well-functioning market, but over the past two decades large industrial loads have mostly filled that role. The new rule enables a wider array of loads to provide priceresponsive demand. FERC (2020b) said in Order No. 2222 that "removing the barriers to participation by distributed energy resource aggregations will enhance the competitiveness, and in turn the efficiency, of RTO/ISO markets and thereby help to ensure just and reasonable and not unduly discriminatory or preferential rates for wholesale electric services."

\footnotetext{
${ }^{3}$ Situation awareness is "the perception of the elements in the environment within a volume of time and space, the comprehension of their meaning, and the projection of their status in the near future" (Endsley 1988). In the context of power systems, situation awareness refers to the operators' state of knowledge and projection of the state of the system, including, but not limited to, the system frequency, voltage, phase angle measurements, information of generator or line outage if such events occur, and other wide-area stability issues.
} 
Not only have DER technologies become more versatile, but advanced communication and information systems have also made it easier to coordinate simultaneous responses from many DERs. These improvements have increased the ability of DER to provide value through a range of grid services, including:

- Capacity value: avoided capital and associated fixed costs for new generation facilities

- Energy value: DER's own energy generation or energy arbitrage can reduce power plant fuel, operation, maintenance, startup and shutdown costs

- Ancillary service value, including contingency reserves, ramping, and frequency regulation

- Non-wires alternatives: intelligent coordination of load and supply to defer or avoid the capital costs for transmission and distribution upgrades

- Voltage support value (Eid et al. 2016; Frick et al. 2021; Neukomm, Nubbe, and Fares 2019).

A key motivation of Order No. 2222 is that higher levels of variable renewable energy increase the need for power system flexibility and ancillary services, both of which DERs could provide. Through energy shifting, DERs can reduce net load ramps, renewable energy curtailment, and ramping and cycling for natural gas combined cycle plants, leading to overall system operational cost savings (Zhou and Mai 2021). Distributed storage, demand response, and other DERs can shift load or modulate load to provide ancillary services that are essential in maintaining power quality, reliability, and security (MacDonald et al. 2012). In a future where more of the transportation, buildings, and industrial loads are electrified, the available capacity of DERs is expected to increase and they will play a greater role in replacing fossil fuel generators to provide power system flexibility and ancillary services, leading to significant carbon emission reductions (Zhou and Mai 2021).

Another key motivation to better integrate DERs into the wholesale market is their contribution in improving power system reliability, which is particularly valuable under extreme weather conditions. Wang et al. (2017) find that even under the current market construct demand response was critical for improving system reliability in the 2014 polar vortex event in PJM because of its advantages in terms of flexibility and high availability. An analysis of the rotating outages in the California Independent System Operator's (CAISO's) service area during the August 2020 summer heat waves shows a significant portion of emergency demand response resources provided load reduction when being called, but the total amount did not meet what was credited against resource adequacy, raising the need for additional analyses and stakeholder engagement to understand demand response participation (CAISO 2021). New market rules are needed to enable effective deployment and control of DERs to meet the changes in the supply and demand balance and provide fast response to maintain grid stability and reliability. Under such rules, DERs can contribute to the system's overall capability of dealing with external shocks, such as extreme weather events, thus boosting grid reliability (Panteli and Mancarella 2015; J. Wang et al. 2019).

\section{Conclusions and Lessons for International Power Markets}

Distributed energy resources are expected to grow dramatically in numbers and capabilities in the coming decades. As aggregated resources, they would bring new value to the bulk power system. Creating enabling market frameworks for DERs to provide a full range of value can 
enhance grid flexibility and help achieve broader objectives in many countries' power systems. Potential benefits of such frameworks include integrating more variable renewable energy, reducing power system costs (through capacity investment deferral, energy shifting, and ancillary services, etc.), strengthening grid reliability, and developing greater energy independence. Such frameworks would also help realize the potential value of the smart meter infrastructure and new sensors and controls as well as information technology.

As more distributed energy resources emerge in international power systems, FERC Order No. 2222 provides useful guidelines for creating a market framework for DER aggregations by:

- Maintaining market neutrality by allowing all types of existing and new DER technologies

- Enhancing market competition by opening the market to the widest possible range of aggregation methods

- Ensuring smooth operation and reliability with data, metering, and telemetry requirements, and coordination with relevant stakeholders. 


\section{References}

CAISO. 2021. "Root Cause Analysis: Mid-August 2020 Extreme Heat Wave." Folsom, CA.

EIA. 2020. "Battery Storage in the United States: An Update on Market Trends." Washington, DC.

Eid, Cherrelle, Paul Codani, Yannick Perez, Javier Reneses, and Rudi Hakvoort. 2016. "Managing Electric Flexibility from Distributed Energy Resources: A Review of Incentives for Market Design." Renewable and Sustainable Energy Reviews. Elsevier Ltd. https://doi.org/10.1016/j.rser.2016.06.008.

Endsley, Mica R. 1988. "Design and Evaluation for Situation Awareness Enhancement." Proceedings of the Human Factors Society Annual Meeting 32 (2): 97-101. https://doi.org/10.1177/154193128803200221.

Feldman, David, and Robert Margolis. 2021. "H2 2020: Solar Industry Update.” Golden, CO. https://doi.org/10.2172/1784533.

FERC. 2020a. "2019 Assessment of Demand Response and Advanced Metering."

— 2020b. "Participation of Distributed Energy Resource Aggregations in Markets Operated by Regional Transmission Organizations and Independent System Operators." Docket No. RM18-9-000; Order No. 2222. Washington, DC.

Frick, Natalie Mims, Snuller Price, Lisa Schwartz, Nichole Hanus, and Ben Shapiro. 2021. "Locational Value of Distributed Energy Resources." Berkeley, CA. https://doi.org/10.2172/1765585.

Gerke, Brian, Giulia Gallo, and Sarah Smith. 2020. "The California Demand Response Potential Study, Phase 3: Final Report on the Shift Resource through 2030.” Berkeley, CA. https://doi.org/10.20357/B7MS40.

Horowitz, Kelsey, Zac Peterson, Michael Coddington, Fei Ding, Ben Sigrin, Danish Saleem, Sara E Baldwin, et al. 2019. "An Overview of Distributed Energy Resource (DER) Interconnection: Current Practices and Emerging Solutions." Golden, CO.

ISO-NE. 2020. “2020 Regional Electricity Outlook.” Holyoke, MA.

MacDonald, Jason S, Peter Cappers, Duncan S Callaway, and Sila Kiliccote. 2012. "Demand Response Providing Ancillary Services A Comparison of Opportunities and Challenges in the US Wholesale Markets." Berkeley, CA.

Mai, Trieu, Paige Jadun, Jeffrey Logan, Colin Mcmillan, Matteo Muratori, Daniel Steinberg, Laura Vimmerstedt, Ryan Jones, Benjamin Haley, and Brent Nelson. 2018. "Electrification Futures Study: Scenarios of Electric Technology Adoption and Power Consumption for the United States." Golden, CO. https://doi.org/10.2172/1459351. 
McGranaghan, Mark, Doug Houseman, Laurent Schmitt, Frances Cleveland, and Eric Lambert. 2016. "Enabling the Integrated Grid: Leveraging Data to Integrate Distributed Resources and Customers." IEEE Power and Energy Magazine 14 (1): 83-93. https://doi.org/10.1109/MPE.2015.2485898.

Neukomm, Monica, Valerie Nubbe, and Robert Fares. 2019. "Grid-Interactive Efficient Buildings Technical Report Series: Overview of Research Challenges and Gaps." Washington, DC. https://doi.org/10.2172/1580214.

Panteli, Mathaios, and Pierluigi Mancarella. 2015. "Influence of Extreme Weather and Climate Change on the Resilience of Power Systems: Impacts and Possible Mitigation Strategies." Electric Power Systems Research. Elsevier Ltd. https://doi.org/10.1016/j.epsr.2015.06.012.

Wang, Fei, Hanchen Xu, Ti Xu, Kangping Li, Miadreza Shafie-khah, and João P.S. Catalão. 2017. "The Values of Market-Based Demand Response on Improving Power System Reliability under Extreme Circumstances.” Applied Energy 193 (May): 220-31. https://doi.org/10.1016/j.apenergy.2017.01.103.

Wang, Jianxiao, Junjie Qin, Haiwang Zhong, Ram Rajagopal, Qing Xia, and Chongqing Kang. 2019. "Reliability Value of Distributed Solar+Storage Systems Amidst Rare Weather Events." IEEE Transactions on Smart Grid 10 (4): 4476-86. https://doi.org/10.1109/TSG.2018.2861227.

Wood Mackenzie. 2020. "United States Distributed Energy Resources Outlook: DER Installations and Forecasts 2016-2025E."

Zhou, Ella, and Trieu Mai. 2021. "Electrification Futures Study: Operational Analysis of U.S. Power Systems with Increased Electrification and Demand-Side Flexibility." Golden, CO. https://doi.org/10.2172/1785329. 